\title{
Correlated Gaussian method for dilute bosonic systems
}

\author{
H. H. Sørensen*, D. V. Fedorov* and A. S. Jensen* \\ *Department of Physics and Astronomy, University of Aarhus, Denmark
}

\begin{abstract}
The weakly interacting trapped Bose gases have been customarily described using the mean-field approximation in the form of the Gross-Pitaevskii equation. The mean-field approximation, however, has certain limitations, in particular it can not describe correlations between particles. We introduce here an alternative variational approach, based on the correlated Gaussian method, which in its simplest form is as fast and simple as the mean-field approximation, but which allows successive improvements of the trial wave-function by including correlations between particles.
\end{abstract}

\section{INTRODUCTION}

Dilute Bose systems trapped in external fields have been a rapidly developing field since the Bose-Einstein condensation was observed almost a decade ago. Theoretically the mean-field approach in the form of the Gross-Pitaevskii equation [1] has been widely and successfully applied to these systems. The computational complexity of the method, and thus the computational time, is independent of the number of particles $N$, in other words it is of the order of $O(1)$.Therefore the method can be applied for large (mesoscopic) bosonic systems, especially when combined with a pseudo-potential (in the form of the $\delta$-function potential) approximation for the interaction potential between particles.

However, the mean-field method has certain limitations, in particular it cannot be easily extended to include correlations between particles. Correlations become important for systems with higher densities and/or stronger effective interactions. Such strong interacting regimes, where the mean-field theory breaks down [2], are now routinely achieved experimentally by use of Feshbach resonances.

Rigorous many-body methods, like the diffusion Monte-Carlo method [2, 3], which include all correlations, has computational complexity of the order $O\left(N^{2}\right)$ and therefore can only be applied for smaller systems. Again, for relatively dilute gases only few simple types of correlation are expected to be important, and including the full machinery of rigorous few-body methods is perhaps by far an overkill for these systems.

Recently, several methods with computational complexity $O(1)$ have been proposed for finite-range [4, 6], and zerorange [7] interactions, where the trial wave-function can incorporate two-body correlations. However, these methods can not be easily extended to include higher order correlations.

We introduce here yet another approach which has an important advantage over the existing methods. Namely the approach can incorporate any desired number and type of correlations - from an uncorrelated wave-function with computational complexity of $O(1)$ at one end, to fully correlated wave-function with computational complexity of $O\left(N^{2}\right)$ and higher at the other end. Thus, depending upon the problem at hand one has a possibility to negotiate a reasonable trade off between the sophistication of the trial wave-function and the computational time.

For dilute gases only few types of lowest order correlations should be of importance, and it turns out that for these types of correlations the method is yet of $O(1)$ order of complexity.

The method is based on correlated Gaussians and amount to a judicious choice of the nonlinear parameters of the basis Gaussians.

\section{METHOD}

\section{Jacobi coordinates}

Consider a system of $N$ particles with masses $m_{i}$, coordinates $\mathbf{r}_{i}, i=1 . . N$, and the Hamiltonian

$$
H=-\sum_{i=1}^{N} \frac{\hbar^{2}}{2 m_{i}} \frac{\partial^{2}}{\partial \mathbf{r}_{i}^{2}}+\sum_{i<j} V_{i j}\left(\mathbf{r}_{i}-\mathbf{r}_{j}\right)+V_{e x t},
$$


where $V_{i j}$ is the potential between particles $i$ and $j$ and $V_{e x t}$ is the external confining potential (a trap). Usually the trap is assumed to be harmonic,

$$
V_{\text {ext }}=\sum_{i=1}^{N} \frac{1}{2} m_{i} \omega^{2} r_{i}^{2}
$$

It is of advantage to introduce the scaled coordinates, $\mathbf{q}_{i}=\sqrt{\frac{m_{i}}{m}} \mathbf{r}_{i}$, where $m$ is an arbitrary mass scale. Indeed the kinetic energy operator $T$ and also the harmonic trap potential $V_{\text {ext }}$ have then a more symmetric form,

$$
T=-\frac{\hbar^{2}}{2 m} \sum_{i} \frac{\partial^{2}}{\partial \mathbf{q}_{i}^{2}}, V_{e x t}=\frac{1}{2} m \omega^{2} \sum_{i} \mathbf{q}_{i}^{2}
$$

The Jacobian of the transformation from $\mathbf{r}$ to the scaled coordinates $\mathbf{q}$ is equal to

$$
\frac{\partial\left(\mathbf{q}_{1} . . \mathbf{q}_{N}\right)}{\partial\left(\mathbf{r}_{1} . . \mathbf{r}_{N}\right)}=\prod_{i}\left(\frac{m_{i}}{m}\right)^{3 / 2}
$$

If all particles have the same mass $m$, there is no difference between coordinates $\mathbf{r}$ and $\mathbf{q}$.

One can make a further suitable linear transformation to a new set of coordinates,

$$
\mathbf{x}_{i}=\sum_{j} U_{i j} \mathbf{q}_{j}
$$

or, in matrix notation $\mathbf{x}=U \mathbf{q}$, where the matrix $U$ is independent of $\mathbf{q}$. The new system of coordinates is called Jacobi if i) one of the coordinates, say the $N$ th, is proportional to the center of mass coordinate $\mathbf{R}$ of the system, $\mathbf{x}_{N}=\sqrt{\frac{\sum_{i} m_{i}}{m}} \mathbf{R}$; ii) the other $N-1$ coordinates are translation invariant; and iii) the transformation preserves the "diagonal" form (3) of the kinetic energy operator.

The last property implies that the transformation (5) and also any transformation between different Jacobi coordinates is unitary, $U U^{T}=1$ (where ${ }^{T}$ denotes transposition), with the corresponding Jacobian being equal to unity. The unitarity means that the so-called hyper-radius $\rho$, defined as $\rho^{2} \equiv \sum \mathbf{q}_{i}^{2}$, is invariant under these transformations,

$$
\rho^{2} \equiv \sum_{i} \mathbf{q}_{i}^{2}=\sum_{i} \mathbf{x}_{i}^{2}=\frac{1}{m} \sum_{i} m_{i} \mathbf{r}_{i}^{2}
$$

With Jacobi coordinates the center of mass coordinate decouples and the hyper-radius it therefore often defined without the contribution from the center of mass coordinate $\mathbf{x}_{N}$,

$$
\rho^{2}=\sum_{i<N} \mathbf{x}_{i}^{2}=\sum_{i} \mathbf{q}_{i}^{2}-N \mathbf{R}^{2}
$$

One of the possible choices of the Jacobi coordinates is

$$
\mathbf{x}_{i=1 . . N}=\sqrt{\frac{\mu_{i}}{m}}\left(\mathbf{R}_{i}-\mathbf{r}_{i+1}\right),
$$

where $\mathbf{R}_{i}$ is the coordinate of the center of mass of the first $i$ particles, $\mathbf{r}_{N+1} \equiv 0$, and $\mu_{i}$ is the reduced mass

$$
\mu_{i}=\frac{M_{i} m_{i+1}}{M_{i}+m_{i+1}}
$$

where $M_{i}=\sum_{k=1}^{i} m_{k}$.

In the following we shall only consider identical particles with $m_{i} \equiv m$.

\section{Hyper-radial approximation}

\section{Non-interacting bosons in a harmonic trap}

Let us consider a system of non-interacting bosons in a harmonic trap. This should be a good first approximation to a system of weakly interacting bosons in a trap which is smooth at the bottom and spherically symmetric. 
The ground-state wave-function $\Psi$ of a system of non-interacting bosons is a product

$$
\Psi=\prod_{i} \psi_{0}\left(q_{i}\right),
$$

where $\psi_{0}(q)$ is the lowest ( $s$-wave) single-particle state of the trap. If the trap is harmonic, $\psi_{0}(q)$ is a Gaussian, $\psi_{0}(q) \propto e^{-\frac{1}{2} \alpha_{0} q^{2}}$, where $\alpha_{0}^{-1 / 2}$ is the (scaled) oscillator length, and the ground-state wave-functions simplifies to a single Gaussian depending only on the hyper-radius $\rho$,

$$
\Psi=\prod_{i} \psi_{0}\left(q_{i}\right) \propto \prod_{i} e^{-\frac{1}{2} \alpha_{0} q_{i}^{2}}=e^{-\frac{1}{2} \alpha_{0} \sum_{i} q_{i}^{2}}=e^{-\frac{1}{2} \alpha_{0} \rho^{2}} .
$$

A single Gaussian $e^{-\frac{1}{2} \alpha_{0} \rho^{2}}$ is thus an exact solution for a system of non-interacting bosons in a harmonic trap. Generally speaking a function of hyper-radius will provide an exact solution to the many-body system in cases where the potential energy of the system depends only on the hyper-radius. The harmonic trap is precisely this type of potential.

\section{Weakly interacting bosons}

If the particles in the trap interact only weakly one can assume, following the ideas from the mean-field theory, that the inter-particle interactions will effectively lead to a certain modification of the field. The solution will then be some square-integrable function of hyper-radius, $\Phi_{H R}(\rho)$, which can be represented as a linear combination of, say, $n$ Gaussians,

$$
\Phi_{H R}(\rho)=\sum_{s=1}^{n} C_{s} e^{-\frac{1}{2} \alpha_{s} \rho^{2}}=\sum_{s} \prod_{i} C_{s} e^{-\frac{1}{2} \alpha_{s} q_{i}^{2}}
$$

where $C_{s}$ are variational parameters, and the range parameters $\alpha_{s}(s=1 . . n)$ are assumed to be fixed and chosen to span the necessary functional space. This trial wave-function is called a hyper-radial approximation. In practice the parameters $\alpha_{s}$ are chosen and then optimized in a stochastic procedure using the ideas from the stochastic variational method [9].

\section{Hyper-radial vs. mean-field}

The variational mean-field approach is based on an assumption that a product wave-function can provide a good description of an interacting system. The trial wave-function $\Psi_{M F}$ is taken as a product of single-particle functions $\psi$,

$$
\Psi_{M F}=\prod_{i} \psi\left(q_{i}\right),
$$

where the functional form of $\psi(q)$ is varied to reach the minimum of the expectation value of the Hamiltonian. Assuming that $\psi$ is a square integrable function, one can represent it as a linear combination of Gaussians,

$$
\psi(q)=\sum_{s} c_{s} e^{-\frac{1}{2} \alpha_{s} q^{2}},
$$

where the coefficients $c_{s}$ are the variational parameters. The trial mean-field wave-function then becomes

$$
\Psi_{M F}=\prod_{i} \sum_{s} c_{s} e^{-\frac{1}{2} \alpha_{s} q_{i}^{2}},
$$

which should be compared with the hyper-radial trial wave-function

$$
\Phi_{H R}(\rho)=\sum_{s} \prod_{i} C_{s} e^{-\frac{1}{2} \alpha_{s} q_{i}^{2}} .
$$

The two trial functions (15) and (16) are similar but not equivalent since the sum and the product operators generally do not commute. Note that the hyper-radial variational parameters $C_{s}$ are linear, while the mean-field parameters $c_{s}$ are 
non-linear ${ }^{1}$. In practice, however, as we shall show by numerical calculations, both trial functions give rather similar results.

Both functions are totally symmetric and thus do not require an explicit symmetrization. The computational time for the variational minimization of the Hamiltonian with both functions is independent of the number of particles.

The hyper-radial function has an advantage that the center of mass motion can be easily decoupled by a (unitary) transformation to relative Jacobi coordinates. Again, the mean-field function cannot be easily improved, while the hyper-radial function is only the basis for further improvements.

\section{Correlations}

\section{Two-body correlations}

The correlation between a pair of particles can be described by a basis function in the form

$$
\Phi_{12}=e^{-\frac{1}{2} \alpha \rho^{2}-\frac{1}{2} \beta\left(\mathbf{q}_{1}-\mathbf{q}_{2}\right)^{2}}
$$

where there are now two independent parameters, $\alpha$ and $\beta$. The trial wave-function is then a linear combination of $\Phi_{12}$ 's with different parameters $\alpha$ and $\beta$,

$$
\Psi=\sum_{s, u} C_{s u} e^{-\frac{1}{2} \alpha_{s} \rho^{2}-\frac{1}{2} \beta_{u}\left(\mathbf{q}_{1}-\mathbf{q}_{2}\right)^{2}}
$$

where $C_{s u}$ are linear variational parameters. The nonlinear parameters $\alpha$ and $\beta$ are again chosen and optimized stochastically.

The basis function is no longer automatically symmetric over all permutations. It has to be symmetrized with respect to particles number 1 and 2 and therefore the symmetrization operator, $\hat{S}$, has to be included when calculating matrix elements,

$$
\hat{S} \Phi_{12}=\left(\begin{array}{c}
N \\
2
\end{array}\right)^{-1} \sum_{i j} \Phi_{i j} .
$$

This is the same type of Faddeev-like decomposition of the wave-function as used in [4, 7, 8].

Fortunately, only a finite number of different terms appear in calculations of the matrix elements, and the computational time is therefore still independent of the number of particles. Indeed the kinetic energy and the external field operators are fully symmetric and therefore the explicit symmetrization of the wave-function is not needed for their matrix elements. The matrix element for the inter-particle potentials reduces to a finite number of terms,

$$
\begin{gathered}
\left(\begin{array}{c}
N \\
2
\end{array}\right)\left\langle\Phi_{12}\left|\sum_{i<j} V_{i j} \hat{S}\right| \Phi_{12}\right\rangle= \\
\left\langle\Phi_{12}\left|\left(V_{12}+2(N-2) V_{13}+\frac{(N-2)(N-3)}{2} V_{34}\right)\right| \Phi_{12}\right\rangle \\
+2(N-2)\left\langle\Phi_{12}\right| \\
\left(V_{12}+V_{13}+V_{23}+(N-3)\left(V_{14}+V_{24}+V_{34}\right)+\frac{(N-3)(N-4)}{2} V_{45}\right) \\
\left|\Phi_{13}\right\rangle \\
+\left(\frac{N(N-1)}{2}-1-2(N-2)\right)\left\langle\Phi_{12}\right| \\
\left(V_{12}+4 V_{13}+V_{24}+V_{34}+2(N-4)\left(V_{15}+V_{35}\right)+\frac{(N-4)(N-5)}{2} V_{56}\right) \\
\left|\Phi_{34}\right\rangle
\end{gathered}
$$

Each individual matrix element in this expression is readily calculated using the expression (28) in the appendix. The structure of the expression basically corresponds to that of [8] where hyper-spherical coordinates were used instead

\footnotetext{
1 indeed the Gross-Pitaevskii mean-field equation is non-linear.
} 
of the Jacobi coordinates used here. Hyper-spherical coordinates allow an easy implementation of a powerful hyperspheric adiabatic expansion method but, on the other hand, do not allow an easy implementation of higher order correlations.

\section{Three-body correlations}

The three-body correlations can be accounted for by a basis function of the form

$$
P h i_{123}=e^{-\frac{1}{2} \alpha \rho^{2}-\frac{1}{2} \beta\left(\mathbf{q}_{1}-\mathbf{q}_{2}\right)^{2}-\frac{1}{2} \gamma\left(\mathbf{q}_{1}-\mathbf{q}_{3}\right)^{2}},
$$

where $\alpha, \beta$ and $\gamma$ are independent parameters. The trial wave-function is then a linear combination of $\Phi_{123}$ 's with different parameters $\alpha$ and $\beta$ and $\gamma$,

$$
\Psi=\sum_{s, u, v} C_{s u v} e^{-\frac{1}{2} \alpha_{s} \rho^{2}-\frac{1}{2} \beta_{u}\left(\mathbf{q}_{1}-\mathbf{q}_{2}\right)^{2}-\frac{1}{2} \gamma_{v}\left(\mathbf{q}_{1}-\mathbf{q}_{3}\right)^{2}},
$$

where $C_{s u v}$ are linear variational parameters, and where the nonlinear parameters $\alpha, \beta$ and $\gamma$ are again chosen and optimized stochastically.

This function must be explicitly symmetrized with respect to particles 1,2 , and 3 . This symmetrization again results in a finite number of different terms as it did for two-body correlations. There are in total 34 different terms and it is therefore not practical to write them down here. The computer program can easily catch the identical terms and thus reduce the computational complexity down to the order of $O(1)$, that is, independent of the number of particles.

\section{NUMERICAL ILLUSTRATIONS}

\section{The Bose system}

We use ${ }^{87} \mathrm{Rb}$ condensate parameters corresponding to fixed scattering length $a_{s}=100$ a.u. and trapping frequency $\omega=2 \pi \times 77.87 \mathrm{~Hz}$, and vary the number of atoms $N=10^{1}-10^{4}$. In all cases, the inverse square root of the nonlinear parameters $\beta_{k}$ and $\gamma_{k}$ are optimized from the random value interval $\left[10^{-4} b_{t} ; 10 b_{t}\right]$ (where $b_{t}=\sqrt{\hbar /(m \omega)} \approx 23095$ a.u. is the trap length), while for the parameters $\alpha$ the interval was $\left[b_{t} ; 10^{3} b_{t}\right]$. In practice only one parameter $\alpha_{0}$ was needed to achieve the chosen accuracy goal of three digits on the interaction energy per particle.

The mean-field validity condition, $n a_{s}^{3} \ll 1$, where $n$ is the particle density, is fulfilled for all values of $N$. Therefore the Gross-Pitaevskii results from the literature should be quite accurate and we shall use them as the reference point. The other regime, $n a_{s}^{3} \gg 1$, shall be investigated separately.

\section{Two-body potentials}

We consider only dilute bosonic systems where the properties largely depend upon the low-energy/large-distance properties of the two-body interaction, that is the s-wave scattering length $a_{s}$. In this regime a zero-range pseudopotential given by a delta function,

$$
V_{\delta}(r)=\frac{4 \pi \hbar^{2} a_{s}}{m} \delta(r),
$$

is proven to provide within a mean-field theory a good approximation to the energy of the system. Applying the delta-function interaction with a Hilbert space of a beyond-mean-field theory, however, requires an appropriate renormalization [7]. The physical scattering length in [23) should be substituted by its first-order Born approximation of the given finite-range potential.

We shall use the delta-function potential for calculation with the uncorrelated hyper-radial trial wave-function.

For correlated calculations we shall use four different finite-range potentials of the form

$$
V(r)=V_{0} e^{-r^{2} / b^{2}}+U_{0} e^{-r^{2} / c^{2}},
$$


TABLE 1. The parameters (in atomic units) of the finite-range Gaussian two-body potentials of the form $V(r)=V_{0} e^{-r^{2} / b^{2}}+U_{0} e^{-r^{2} / c^{2}}$ used in the calculations. $N_{b}$ is the number of bound states in the potential. The $s$-wave scattering length $a_{s}$ is equal 100 a.u. for all potentials.

\begin{tabular}{cccccc}
\hline Designation & $b$ & $V_{0}$ & $c$ & $U_{0}$ & $N_{b}$ \\
\hline H (hard) & 58.69 & $1.906 \times 10^{-7}$ & 0 & 0 & 0 \\
S (soft) & 550.0 & $1 \times 10^{-11}$ & 0 & 0 & 0 \\
A (attractive) & 10 & $-1.906 \times 10^{-7}$ & 0 & 0 & 1 \\
W (well) & 4.4 & $5.566 \times 10^{-5}$ & 10 & $-1.125 \times 10^{-6}$ & 1 \\
\hline
\end{tabular}

TABLE 2. The interaction energy per particle, $\frac{E}{N}-\frac{3}{2} \hbar \omega$, where $E$ is the total energy, for the system described in the text. Results are given for the hard-core $(\mathrm{H})$ and softcore $(\mathrm{S})$ potential from Table 1 with different trial wave functions $(1 \mathrm{~b}-$ uncorrelated, $2 \mathrm{~b}$ - two-body correlations, $3 \mathrm{~b}$ - three-body correlations) as well as for the $\delta$-function potential with uncorrelated wave-function. The last column shows the Gross-Pitaevskii (mean-field) results from [10] and [11].

\begin{tabular}{|c|ccc|ccc|c|c|}
\hline & \multicolumn{2}{|c|}{ hard-core potential } & \multicolumn{3}{|c|}{ soft-core potential } & $\delta$-function & \\
$N$ & $1 \mathrm{~b}$ & $2 \mathrm{~b}$ & $3 \mathrm{~b}$ & $1 \mathrm{~b}$ & $2 \mathrm{~b}$ & $3 \mathrm{~b}$ & $1 \mathrm{~b}$ & $\mathrm{GP}$ \\
\hline 10 & .329 & .0155 & .0154 & .0179 & .0154 & .0154 & .0154 & .0154 \\
20 & .599 & .0326 & .0325 & .0373 & .0320 & .0320 & .0320 & .0320 \\
50 & 1.18 & .0832 & .0828 & .0923 & .0795 & .0794 & .0798 & .0792 \\
100 & 1.83 & .165 & .164 & .177 & .153 & .153 & .153 & .151 \\
1000 & 6.29 & 1.32 & 1.32 & 1.09 & 1.00 & .999 & .978 & .930 \\
5000 & 13.2 & 4.48 & 4.47 & 2.88 & 2.75 & 2.75 & 2.64 & 2.45 \\
10000 & 17.8 & 7.27 & 7.26 & 4.15 & 4.02 & 4.02 & 3.83 & 3.58 \\
\hline
\end{tabular}

where the parameters of the potentials are specified in Table 1 The first potential, marked $\mathrm{H}$, is a hard repulsive core, the second, S, is a soft repulsive core, the third, A, is an attractive well, and the fourth, $\mathrm{W}$, is a semi-realistic well with a repulsive core and an attractive pocket. All potentials have the same scattering length, $a_{s}=100$ a.u., and in the dilute regime should therefore provide identical energies if correlations are appropriately included.

\section{Results}

The results are collected in Tables 2 and 3 where we show the interaction energy per particle, $\frac{E}{N}-\frac{3}{2} \hbar \omega$ (where $E$ is the total energy of the system), for different combinations of numbers of particles, potentials, and trial wave-functions. The absence of a number for the attractive and realistic potential means that there are many strongly bound (collapsed) states and an analog of the condensate state located in the trap does not exist.

TABLE 3. The same as Table 2 for the attractive (A), and realistic (W) potentials from Table 1 For larger number of particles and higher correlations the potentials produce a large number of strongly bound (collapsed) states and thus no condensate state could have been traced.

\begin{tabular}{|c|cc|ccc|c|}
\hline & \multicolumn{2}{|c|}{ attractive potential } & \multicolumn{3}{|c|}{ realistic potential } & \\
$N$ & $1 \mathrm{~b}$ & $2 \mathrm{~b}$ & $1 \mathrm{~b}$ & $2 \mathrm{~b}$ & $3 \mathrm{~b}$ & $\mathrm{GP}$ \\
\hline 10 & -.0021 & .0147 & .0383 & .0154 & .0154 & .0154 \\
20 & -.0044 & .0264 & .0599 & .0320 & .0320 & .0320 \\
50 & -.0114 & .0228 & .188 & .0804 & .0802 & .0792 \\
100 & -.0233 & -.0042 & .344 & .156 & .155 & .151 \\
1000 & & & 1.78 & 1.07 & & .930 \\
5000 & & & 4.33 & 3.27 & & 2.45 \\
10000 & & & 6.11 & 5.09 & & 3.58 \\
\hline
\end{tabular}




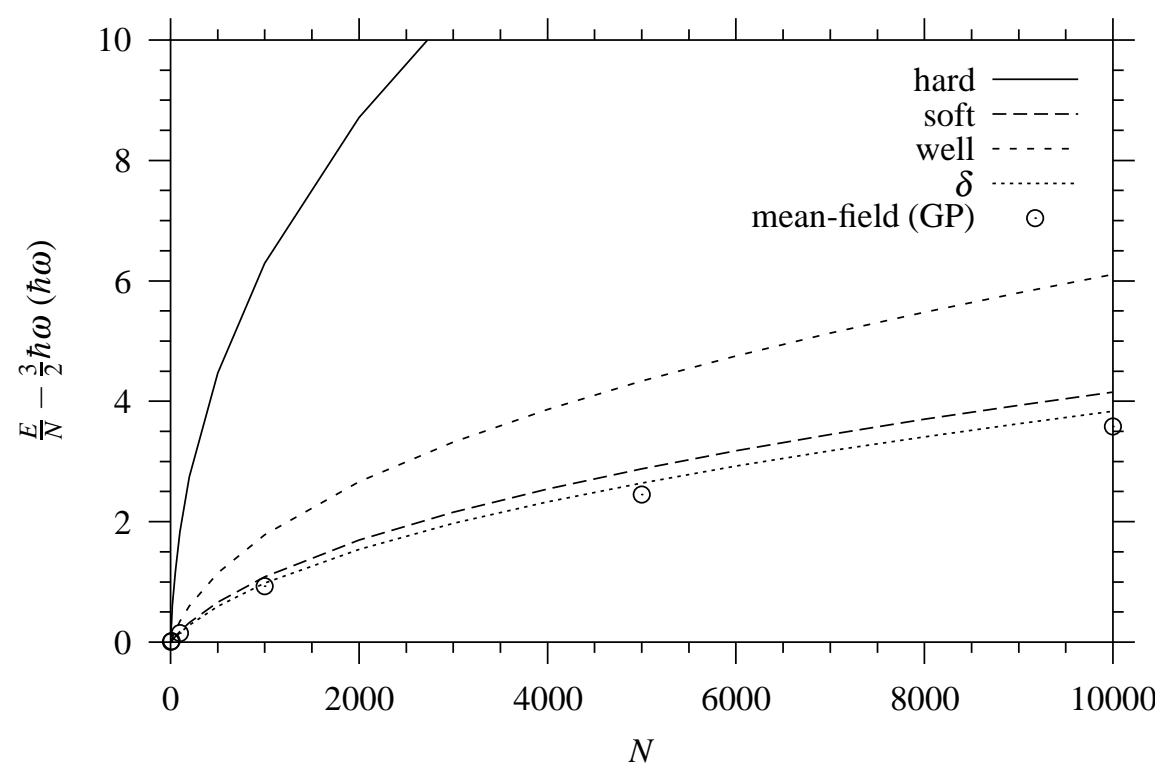

FIGURE 1. Interaction energy per particle as function of the number of particles $N$ for the uncorrelated trial wave-function.

\section{Uncorrelated wave-function}

The results for different potentials with the uncorrelated hyper-radial trial wave-function are given in Tables 23 and also represented on Fig 1

Importantly, the combination of delta-function pseudo-potential with the uncorrelated hyper-radial wave-function give results within a few per cent of the mean-field theory. The pseudo-potential therefore seems to be equally well suited for both mean-field and hyper-radial approximations.

One can use this very fast uncorrelated pseudo-potential approximation to a great effect as a tool to optimize the parameters of the Gaussians to be used in the more demanding correlated calculations with finite-range potentials.

The finite-range potentials show large deviations since the uncorrelated wave-function is not suited for them. The hard-core potential, as could be expected, is especially bad for the uncorrelated wave-function. The attractive potential produces for larger number of particles a strongly bound (collapsed) ground-state and is therefore not shown on the figure.

\section{Two-body correlations}

The results with the two-body correlated trial wave-function are given in Tables 23 and also represented on Fig. 2 Apparently, inclusion of two-body correlations dramatically improves the results. This seems to support the assertion in $[4,7,8]$ that the two-body correlations are of the utmost importance for the dilute gases.

The hard-core potential, although doing much better with the two-body correlated wave-function, is still the farthest off especially for large number of particles. The soft-core potential on the other hand is now very close to the meanfield results.

\section{Three-body correlations}

We do not show a separate figure for the three-body correlations as they turn out not to produce large effects on the energies apart from potentials with attraction, where the three-body correlations quite expectedly straight away lead to a large number of strongly bound (collapsed) states.

Thus, for model repulsive finite-range potentials and dilute systems the three-body correlations are of much less importance that two-body correlations. 


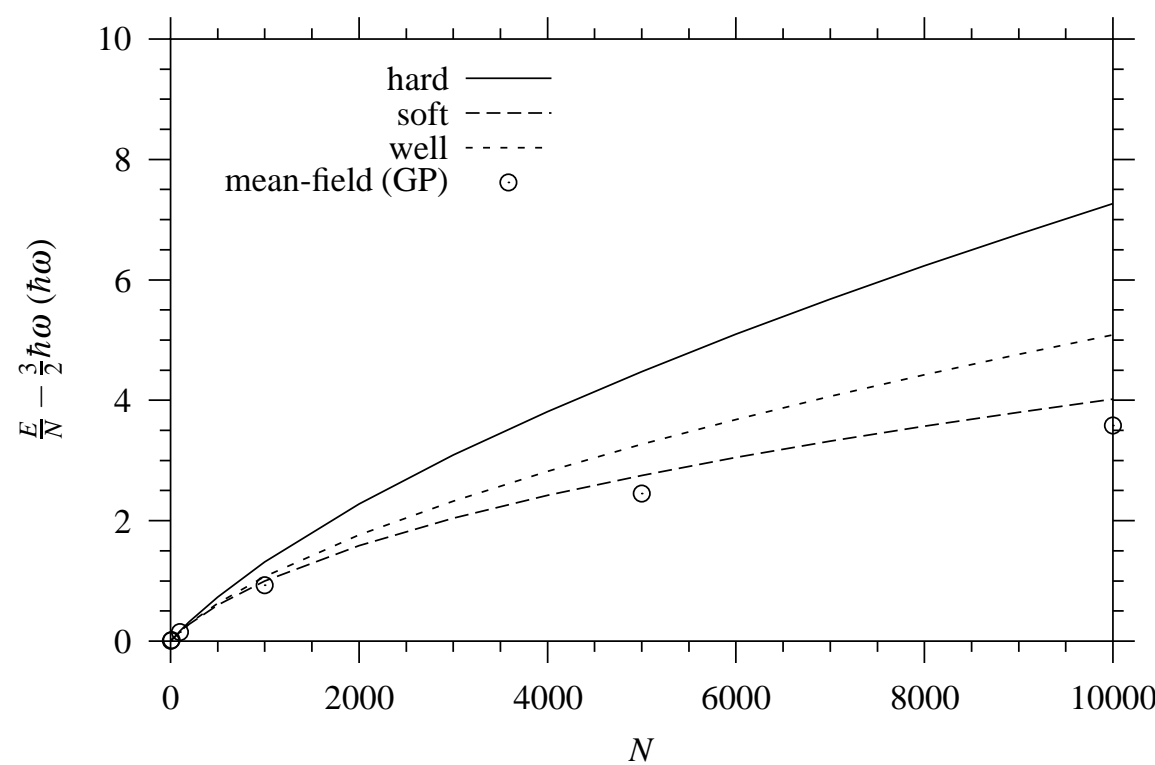

FIGURE 2. Interaction energy per particle as function of the number of particles $N$ for the trial wave-function with two-body correlations.

\section{CONCLUSION}

We have introduced a new approach, based on correlated Gaussian method, to investigate dilute Bose systems. The approach allows to include consecutively correlations of different orders in the trial wave-function. In its lowest (uncorrelated) order with zero-range pseudo-potentials the approach is comparable to the mean-field (Gross-Pitaevskii) theory.

We have performed an exploratory numerical investigation of two- and three-body correlation in a dilute Bose system with different number of particles and different finite-range potentials. For the condensate state the two-body correlations turn out to be by far the most important and suffice to provide a quantitative description of the system with soft-core potentials.

\section{ACKNOWLEDGMENTS}

H. H. Sørensen would like to thank Christoffer Dam Bruun for many interesting discussions and valuable assistance during implementation of the method.

\section{APPENDIX: CORRELATED GAUSSIAN METHOD}

The trial wave-function is represented as a linear combination of correlated Gaussians, $|A\rangle$, which have the form

$$
A=\exp \left(-\frac{1}{2} \sum_{i, j=1}^{N}\left(\mathbf{x}_{i} \cdot \mathbf{x}_{j}\right) A_{i j}\right) \equiv \exp \left(-\frac{1}{2} \mathbf{x}^{T} A \mathbf{x}\right)
$$

where $A$ is a positively definite symmetric matrix and $\mathbf{x}$ is a set of (scaled Jacobi) coordinates. Correlated Gaussians form a full basis since any square-integrable function can be represented as a linear combination of Gaussians with arbitrary precision. The elements of the parameter matrix $A$ can be optimize using the stochastic method [9].

The important matrix elements which are used in the calculations are the overlap of two Gaussians,

$$
\left\langle A \mid A^{\prime}\right\rangle=\left(\frac{(2 \pi)^{N}}{\operatorname{det}\left(A+A^{\prime}\right)}\right)^{3 / 2}
$$


the matrix element of the kinetic energy operator,

$$
\left\langle A\left|-\frac{\hbar^{2}}{2 m} \sum_{i} \frac{\partial^{2}}{\partial \mathbf{x}_{i}^{2}}\right| A^{\prime}\right\rangle=\frac{\hbar^{2}}{2 m} 3 \operatorname{tr}\left(\left(A+A^{\prime}\right)^{-1} A A^{\prime}\right)\left\langle A \mid A^{\prime}\right\rangle,
$$

and the matrix element of the two-body potential $V\left(\mathbf{r}_{i}-\mathbf{r}_{j}\right)$,

$$
\left\langle A\left|V\left(\mathbf{r}_{i}-\mathbf{r}_{j}\right)\right| A^{\prime}\right\rangle=\int_{-\infty}^{+\infty} d^{3} r V(\mathbf{r})\left\langle A\left|\delta\left(b_{i j}^{T} \mathbf{x}-\mathbf{r}\right)\right| A^{\prime}\right\rangle=G_{c_{i j}}[V]\left\langle A \mid A^{\prime}\right\rangle,
$$

where $\mathbf{r}_{i}-\mathbf{r}_{j}=b_{i j}^{T} \mathbf{x}, c_{i j}^{-1}=b_{i j}^{T}\left(A+A^{\prime}\right)^{-1} b_{i j}$, and $G_{c}[V]$ is the Gaussian transform of the potential

$$
G_{c}[V]=\left(\frac{c}{2 \pi}\right)^{3 / 2} \int d^{3} r V(\mathbf{r}) e^{-\frac{1}{2} c r^{2}}
$$

Other useful integrals

$$
\left\langle A\left|\mathbf{x}^{T} B \mathbf{x}\right| A^{\prime}\right\rangle=3 \operatorname{tr}\left(\left(A+A^{\prime}\right)^{-1} B\right)\left\langle A \mid A^{\prime}\right\rangle
$$

$$
\begin{aligned}
\left\langle A\left|\delta\left(\mathbf{b}^{T} \mathbf{x}-\mathbf{q}\right)\right| A^{\prime}\right\rangle=\left(\frac{\beta}{2 \pi}\right)^{3 / 2} e^{-\frac{1}{2} \beta r^{2}}\left\langle A \mid A^{\prime}\right\rangle, \text { where } \beta^{-1}=\mathbf{b}^{T}\left(A+A^{\prime}\right)^{-1} \mathbf{b} & \\
G_{c}\left[\frac{1}{r}\right] & =2 \sqrt{\frac{c}{2 \pi}} \\
G_{c}[\delta(r)] & =\left(\frac{c}{2 \pi}\right)^{3 / 2} ; \\
G_{c}\left[e^{-\frac{1}{2} k r^{2}}\right] & =\left(\frac{c}{c+k}\right)^{3 / 2}
\end{aligned}
$$

\section{REFERENCES}

1. L. Pitaevskii, and S. Stringari, Bose-Einstein Condensation, Clarendon Press, Oxford, 2003.

2. D. Blume, and C.H. Greene, Phys. Rev. A63, 063601 (2001).

3. J.L. DuBois and H.R. Glyde, Phys.Rev. A68, 033602 (2003).

4. O. Sørensen, D.V. Fedorov, and A.S. Jensen, Few-Body Systems, 34, 203 (2004).

5. H.H. Sørensen, Master Thesis, Department of Physics and Astronomy, University of Aarhus, 2004, arxiv.org/condmat/0502126.

6. T.K. Das, and B. Chakrabarti, arxiv.org/quant-ph/0408061.

7. T. Sogo, O. Sørensen, A.S. Jensen, and D.V. Fedorov, arxiv.org/cond-mat/0407065.

8. O. Sørensen, D.V. Fedorov, and A.S. Jensen, J. Phys. B37, 93 (2004).

9. Y. Suzuki and K. Varga, Stochastic Variational Approach to Quantum-Mechanical Few-Body Problems, Springer-Verlag Berlin Heidelberg, 1998.

10. B.A. McKinney, M. Dunn, and D.K. Watson, Phys. Rev., \{A69\}, 053611 (2004).

11. A.Fabrocini, and A.Polls, Phys.Rev. A60, 2319 (1999) 